\title{
Crystal structure of HutZ, a heme storage protein from Vibrio cholerae: A structural mismatch observed in the region of high sequence conservation
}

\author{
Xiuhua Liu' ${ }^{1,3+}$, Jing Gong ${ }^{2 \dagger}$, Tiandi Wei ${ }^{1}$, Zhi Wang ${ }^{1}$, Qian Du', Deyu Zhu', Yan Huang ${ }^{1}$, Sujuan Xu' \\ and Lichuan $\mathrm{Gu}^{1 *}$
}

\begin{abstract}
Background: HutZ is the sole heme storage protein identified in the pathogenic bacterium Vibrio cholerae and is required for optimal heme utilization. However, no heme oxygenase activity has been observed with this protein. Thus far, HutZ's structure and heme-binding mechanism are unknown.

Results: We report the first crystal structure of HutZ in a homodimer determined at $2.0 \AA$ resolution. The HutZ structure adopted a typical split-barrel fold. Through a docking study and site-directed mutagenesis, a heme-binding model for the HutZ dimer is proposed. Very interestingly, structural superimposition of HutZ and its homologous protein HugZ, a heme oxygenase from Helicobacter pylori, exhibited a structural mismatch of one amino acid residue in $\beta 6$ of HutZ, although residues involved in this region are highly conserved in both proteins. Derived homologous models of different single point variants with model evaluations suggested that Pro ${ }^{140}$ of HutZ, corresponding to Phe ${ }^{215}$ of HugZ, might have been the main contributor to the structural mismatch. This mismatch initiates more divergent structural characteristics towards their C-terminal regions, which are essential features for the heme-binding of HugZ as a heme oxygenase.
\end{abstract}

Conclusions: HutZ's deficiency in heme oxygenase activity might derive from its residue shift relative to the heme oxygenase HugZ. This residue shift also emphasized a limitation of the traditional template selection criterion for homology modeling.

Keywords: HutZ, Heme-binding, Crystal structure, Homology modeling

\section{Background}

Iron is an essential element for the Gram-negative pathogenic bacterium Vibrio cholerae. It plays important roles in the microbe's survival and its ability to cause the diarrheal disease cholera of $V$. cholerae. Nevertheless, the concentration of free iron is extremely low in the environment as well as in the human hosts. Under iron starvation conditions, $V$. cholerae has evolved several high-affinity iron uptake systems [1]. Synthesis and secretion of the catechol-type siderophore vibriobactin is

\footnotetext{
* Correspondence: Icgu@sdu.edu.cn

${ }^{\dagger}$ Equal contributors

'State Key Laboratory of Microbial Technology, School of Life Sciences, Shandong University, Jinan 250100, China

Full list of author information is available at the end of the article
}

the main mechanism for obtaining iron [2]. Siderophores, such as schizokinen [3], enterobactin [4,5] and ferrichrome [6], produced by other microorganisms, can also be utilized by $V$. cholerae.

Heme, an excellent iron source in the environment and human hosts, can be used by $V$. cholerae in the free form or with heme-binding proteins $[7,8]$. It is first transported into the cell with the assistance of the corresponding TonB-dependent outer membrane receptors and ATP-binding cassette transporter system proteins $[3,9]$, and then the iron released from heme by cytoplasmic heme oxygenase.

However, to date, no heme oxygenase has been reported in $V$. cholerae. BLAST searches against the NCBI database

\section{Ciomed Central}


[10] have also returned no heme oxygenase homologues for $V$. cholerae. Therefore, the fate of heme after it enters $V$. cholerae's cytoplasm remains mysterious, and little is known regarding which proteins contribute to heme utilization in the cytoplasm. In 2004, Wyckoff and coworkers identified in $V$. cholerae the heme-binding protein HutZ, a protein essential for the optimal utilization of heme as an iron source [11]. However, no heme oxygenase activity has been observed for this protein, which indicates that HutZ serves only as a heme storage protein [11]. NCBI BLAST searches have shown that HutZ belongs to the pyridoxine-5'-phosphate (PNP) oxidaselike superfamily and shares $35 \%$ sequence identity with the heme oxygenases HugZ from Helicobacter pylori $[12,13]$ and ChuZ from Campylobacter jejuni [14]. Meanwhile, sequence comparisons have shown that HutZ shares low sequence identities ( 10\%) with other known representative heme oxygenases, such as $\mathrm{HmuO}$ from Corynebacterium diphtheria [15], HemO from Neisseria meningitidis [16], IsdG from Bacillus anthracis [17], and ChuS from Escherichia coli O157:H7 [18].

To gain further insight into the mechanism of the mysterious function of HutZ, this protein from $V$. cholerae (strain N16961) was overexpressed and its crystal structure determined at $2.0 \AA$ resolution. The HutZ structure appeared to a typical split-barrel fold that is usually conserved in FMN-binding proteins. As heme did not cocrystallize with HutZ, molecular docking and site-directed mutation experiments were performed to investigate the interaction mechanism of HutZ and heme.

In the absence of experimentally solved protein structures, homology modeling is widely used to predict a structure based on one or more known structures of homologous proteins. Such a model is developed based on the general rule that similar protein sequences correspond to similar protein structures. Notably, in the comparison of the structures of HutZ and HugZ, a structural mismatch was identified in one amino acid residue after the corner of $\beta 6$ of HutZ, where both protein sequences are identical except for two pairs of different amino acid residues. This observation suggested a potential hazard in the accuracy or application of the homology modeling method, in particular in the accuracy of the input sequence alignment. In the light of this, a series of homologous models were constructed to further verify the sequence-structure relationship between HutZ and HugZ and to explore functional implications from the HutZ structure. As ChuZ and HugZ share high sequence identity (53\%) and structural similarity (root mean square deviation (RMSD) of $2.1 \AA$ for the protein backbone), HugZ was employed as the representative heme oxygenase for homology modeling of different HutZ variants and for structural comparisons.

\section{Results}

\section{The overall structure of HutZ}

The structure of HutZ contains four monomer molecules (A, B, C and D) in an asymmetric unit that form two homodimers ( $\mathrm{AB}$ and $\mathrm{CD}$ ) (Figure 1A). Each monomer includes amino acid residues 13-150 of the entire HutZ protein (residues 1-176). Protein interface analysis with CCP4i showed that the interface area between monomers A and B was 1575.1 $\AA^{2}$ and 1569.4 $\AA^{2}$ for $C$ and $D$, covering approximately $18 \%$ of the total solvent accessible surface area of $17208 \AA^{2}$. This suggested that the homodimer forms, which represent the aggregation state of most split-barrel proteins in solution, were stable for HutZ. The existence of HutZ homodimers in solution was further confirmed by gel filtration chromatography on a Superdex-200 column (Additional file 1).

Although the HutZ homodimer adopted a typical split-barrel fold that is commonly conserved in FMNbinding proteins, no such activity was observed for HutZ (data not shown). Superimposition of both monomers from each dimer produces an RMSD of $0.294 \AA$ for all corresponding $\mathrm{C} \alpha$ atoms. A HutZ monomer consisted of four $\alpha$-helices interwoven with six $\beta$-strands (Figure 1B), a structure similar to the $\mathrm{C}$-terminal domain of $\mathrm{HugZ}$ (PDB code: 3GAS) (Figure 2) [13]. Six antiparallel strands, $\beta 1-\beta 6$, produced a $\beta$-barrel of a Greek key topology (Additional file 2). Helices $\alpha 1$ and $\alpha 2$ were packed against the $\beta$-barrel and blocked each opening of the $\beta$-barrel. Helices $\alpha 3$ and $\alpha 4$ were loaded on one side of the $\beta$-barrel, with the HutZ dimer bound mainly through interactions of exposed $\beta$-barrel from each monomer. The two $\beta$-barrels were packed with each other side by side, bringing together surfaces distant from helices $\alpha 3$ and $\alpha 4$ on the loading side.

\section{Heme-binding site}

A number of approaches were attempted to produce crystals of HutZ-heme complex, but no crystals were obtained. In this case, we carried out molecular docking to investigate the interactions between heme and HutZ. There are two large clefts in the HutZ dimer interfaces that are postulated, due to similarities with HugZ, to be the heme-binding pocket. The first-ranked resulting model of HutZ dimer-heme complex showed that the main contributors to the heme coordination included $\mathrm{His}^{63}$ loaded at the helix $\alpha 2$ of one monomer and $\mathrm{Arg}^{92}$ originating from strand $\beta 5$ of the other (Figure $3 \mathrm{~A}$ ). These two residues cooperatively coordinated the iron atom in the center of the heme molecule, with the distance between $\mathrm{His}^{63}$ and the iron atom at approximately $3.6 \AA$ and between $\mathrm{Arg}^{92}$ and the iron atom at $3.9 \AA$. UV absorption spectral analysis on native and mutant forms of HutZ-heme complexes were performed to 

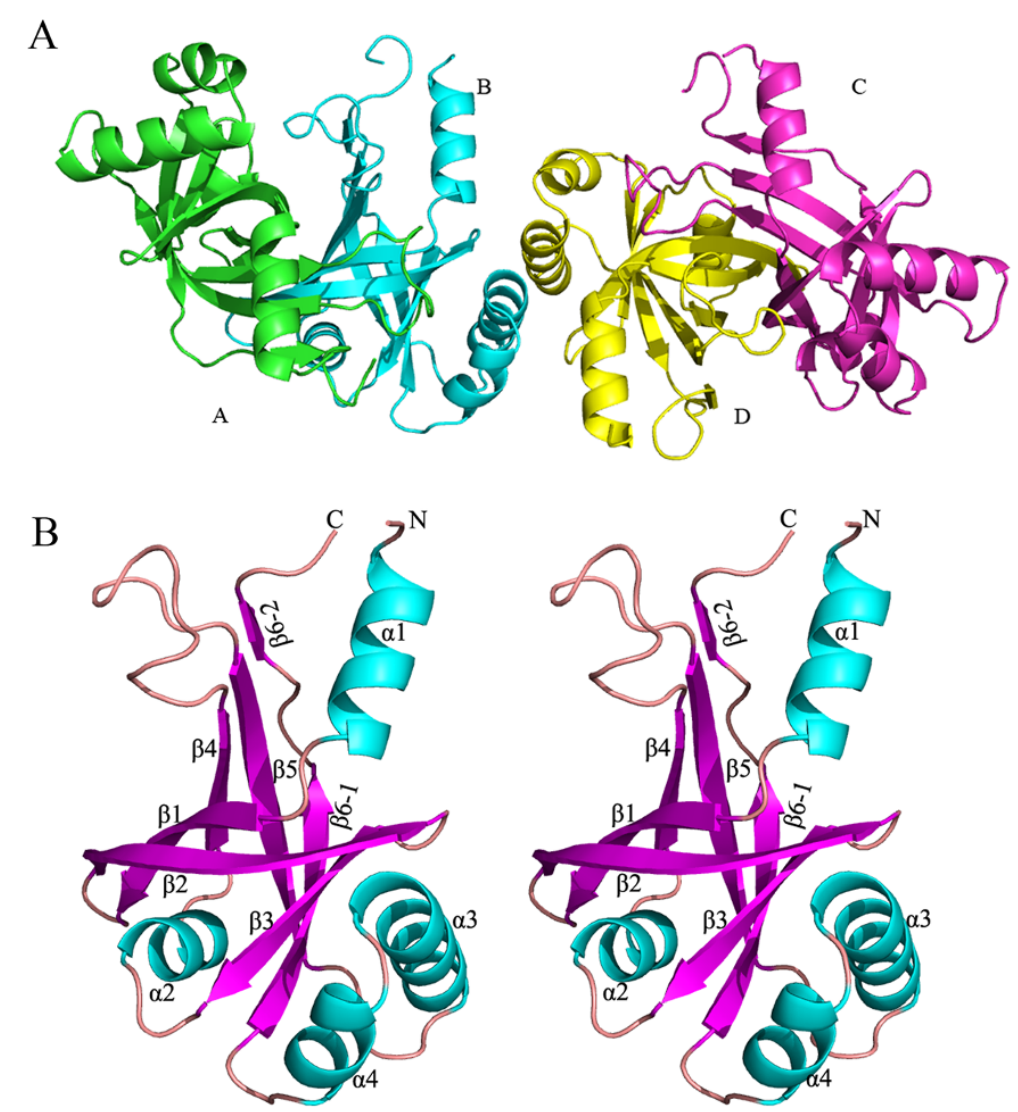

Figure 1 Overall structure of HutZ (A) and stereoview of HutZ monomer (B). (A) There are two dimers in an asymmetric unit, termed A (green), B (cyan), C (magenta) and D (yellow) for clarification. (B) Rainbow-colored scheme representation of HutZ monomer. Four a-helices and six $\beta$-strands are labeled.

explore whether $\mathrm{His}^{63}$ and $\mathrm{Arg}^{92}$ were both necessary for heme-binding. The Soret peak for the native HutZ-heme complex was at $410 \mathrm{~nm}$; for the single mutant H63A reconstituted with heme, it was slightly shifted to $420 \mathrm{~nm}$, indicating that $\mathrm{His}^{63}$ slightly affected heme binding. For R92A, the Soret band was not altered in comparison with the native HutZ-heme complex (Figure 3B), suggesting that $\mathrm{Arg}^{92}$ did not affect heme binding. However, there was no characteristic Soret peak detected for the double point mutant H63A-R92A reconstituted with heme. The Soret peak data collected were from full length HutZ and truncated HutZ (data not shown for full length HutZ). These observations indicated that at least one of these residues, $\mathrm{His}^{63}$ and $\mathrm{Arg}^{92}$, must exist to effectively coordinate the iron atom in heme, and either of them is sufficient. Additionally, eleven residues from monomer A $\left(\mathrm{Ser}^{42}, \mathrm{Tyr}^{43}, \mathrm{Pro}^{45}, \mathrm{Ser}^{58}, \mathrm{Ile}^{60}, \mathrm{Ala}^{61}, \mathrm{Arg}^{62}, \mathrm{Arg}^{65}, \mathrm{Leu}^{127}\right.$, $\mathrm{Asp}^{132}$ and $\mathrm{Phe}^{133}$ ) and three residues from monomer B $\left(\mathrm{Phe}^{88}{ }^{8} \mathrm{Thr}^{94}\right.$ and $\mathrm{Phe}^{145}$ ) around the heme-binding cleft participated in stabilizing the heme. These residues were fully conserved or replaced with similar residues in comparison with the residues stabilizing the heme group in the HugZ-heme complex (Additional file 3).
Structural comparison between HutZ and HugZ

Structural superimposition revealed that the HutZ monomer shared high structural similarity with HugZ monomer (structure RMSD $=1.645 \AA$ and sequence identity $=35 \%$ ), which is a heme oxygenase from Helicobacter pylori $[12,13]$. Sequence alignment showed that the HugZ Nterminal domain (residues 1-80) was absent in HutZ, and the C-terminal loop (residues 238-249) of HugZ was variable in HutZ. In HutZ, the $\beta 6$ contained a four-residuelong corner structure $\left(\operatorname{Pro}^{140}-\right.$ Gly $^{143}$ ) that divides $\beta 6$ into two segments (Figure 1B), designated as $\beta 6-1$ $\left(\mathrm{Phe}^{133}-\mathrm{Gln}^{139}\right.$ ) and $\beta 6-2\left(\mathrm{Leu}^{144}-\mathrm{Gly}^{148}\right.$ ) respectively, while the corresponding $\beta 9$ in HugZ is also divided into two segments $\beta 9-1$ ( $\mathrm{Phe}^{208}-\mathrm{Asp}^{214}$ ) and $\beta 9-2\left(\mathrm{Gly}^{218}\right.$. $\mathrm{Gly}^{223}$ ) by a three-residue-long corner $\left(\mathrm{Phe}^{215}-\mathrm{Glu}^{217}\right.$ ) (Figure 4A). The Fo_Fc omit map clearly revealed the positions of the four amino acid residues composing the corner of $\beta 6$ in HutZ (Additional file 4). Very interestingly, although the segment $\mathrm{Gly}^{143}-\mathrm{Tyr}^{153}$ in HutZ was identical to the segment $\mathrm{Gly}^{218}-\mathrm{Tyr}^{228}$ in HugZ except for two pairs of amino acid residues ( $\mathrm{Arg}^{219}$ of HutZ and Leu ${ }^{144}$ of HugZ, Phe ${ }^{225}$ of HutZ and Gly ${ }^{150}$ of HugZ) (Figure 4A), these residues did not match each other in one-to-one 


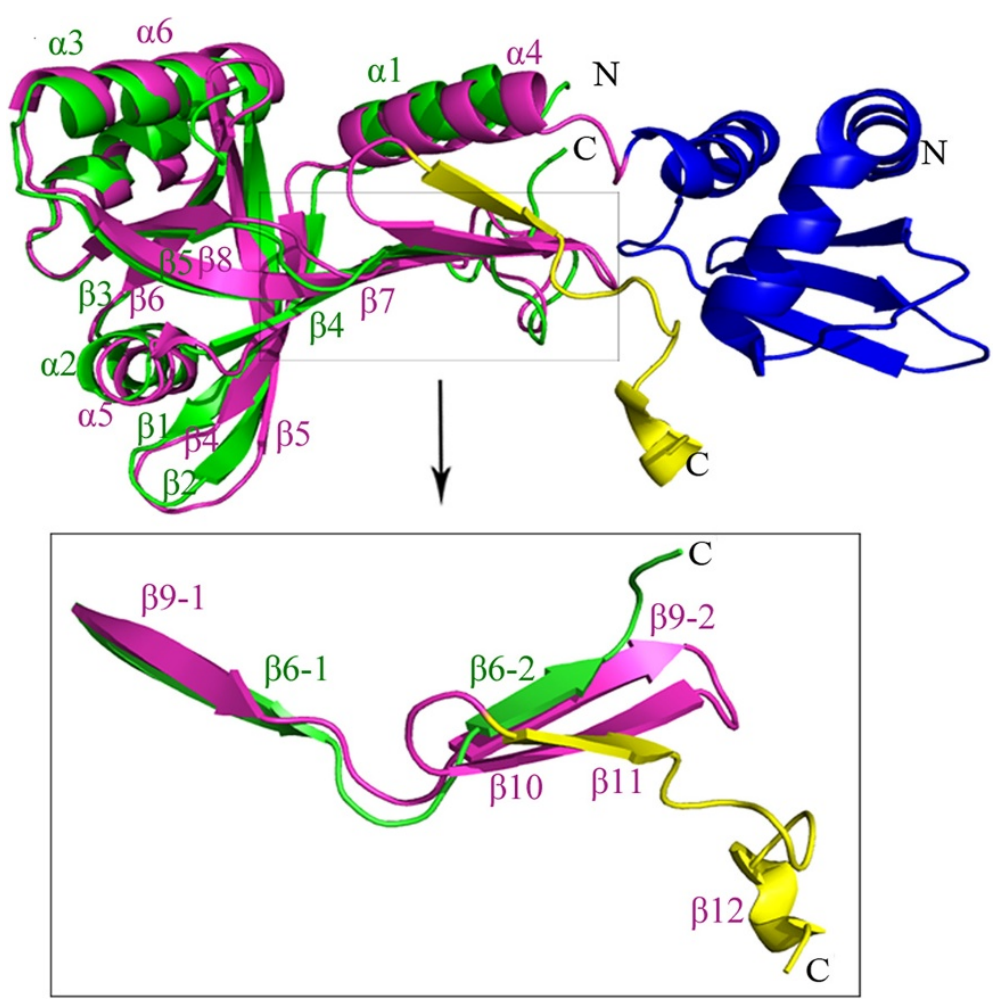

Figure 2 Structure comparison of HutZ and HugZ monomers. Overall HutZ monomer folding (green) is very similar to the homologous protein HugZ (magenta). HugZ N-terminal domain which is absent in HutZ is labeled in blue, and HugZ variable C-terminal region is in yellow. Secondary structural assignment for HutZ was labeled in green and HugZ in magenta.

correspondence in the superimposition of threedimensional structures (Figure $4 \mathrm{~B}$ and $\mathrm{C}$ ). Rather, $\beta 6-2$ was shifted frontward by one amino acid residue relative to $39-2$. For example, $\mathrm{Phe}^{145}$ of HutZ did not match Phe $^{220}$ of HugZ, but corresponded to the preceding $\mathrm{Arg}^{219}$. This structural mismatch was substantially represented as changes in hydrogen bonding patterns between adjacent $\beta$-strands. Two adjacent $\beta$-strands form a hydrogen bond network in which the $\mathrm{N}-\mathrm{H}$ groups or $\mathrm{C}=\mathrm{O}$ groups of one strand establish hydrogen bonds with the $\mathrm{C}=\mathrm{O}$ groups or $\mathrm{N}-\mathrm{H}$ groups of another. Normally, there are two hydrogen bonds ( $\mathrm{N}-\mathrm{H}-\mathrm{O}$ and $\mathrm{O}-\mathrm{H}-\mathrm{N})$ on every second amino acid residue in one strand. In the HutZ and HugZ structures, the hydrogen bonding patterns were both regular on $\beta 6-1$ and $\beta 9-1$ (Additional file 5. A and B). However, in the four-residue-long corner of HutZ, no hydrogen

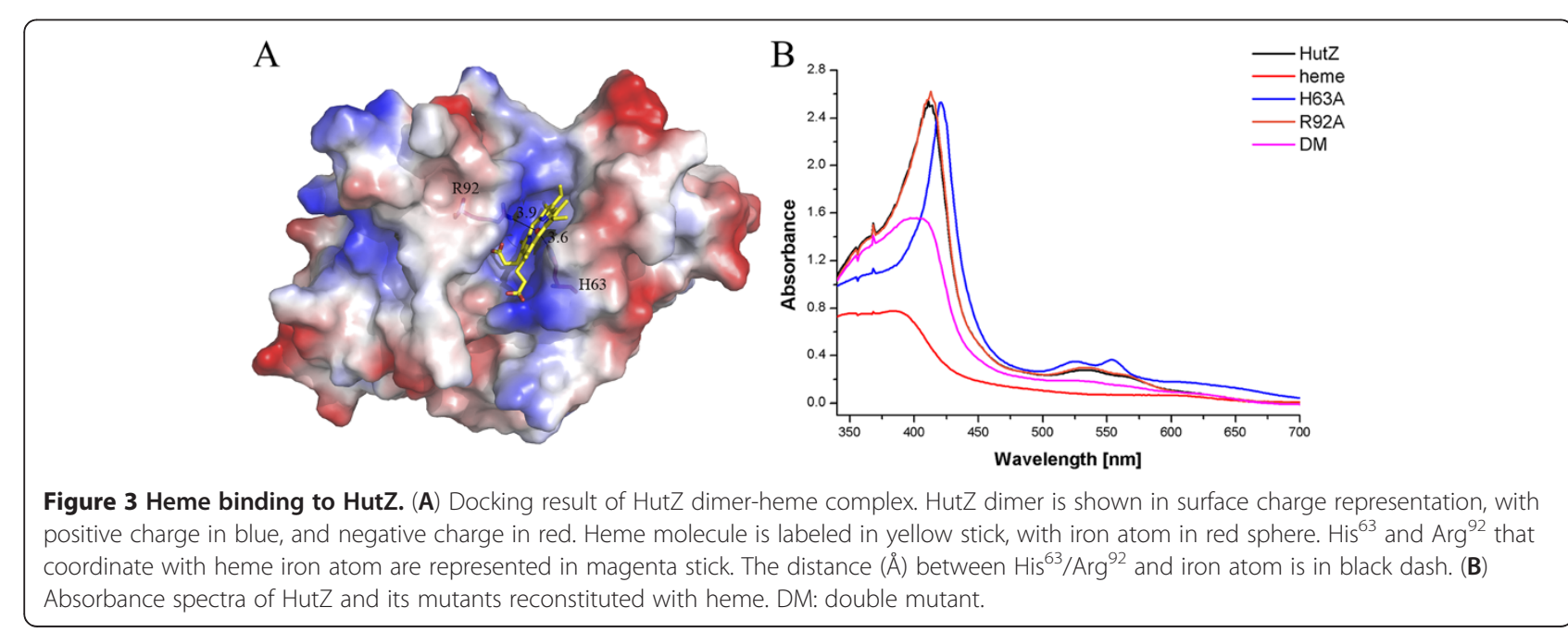




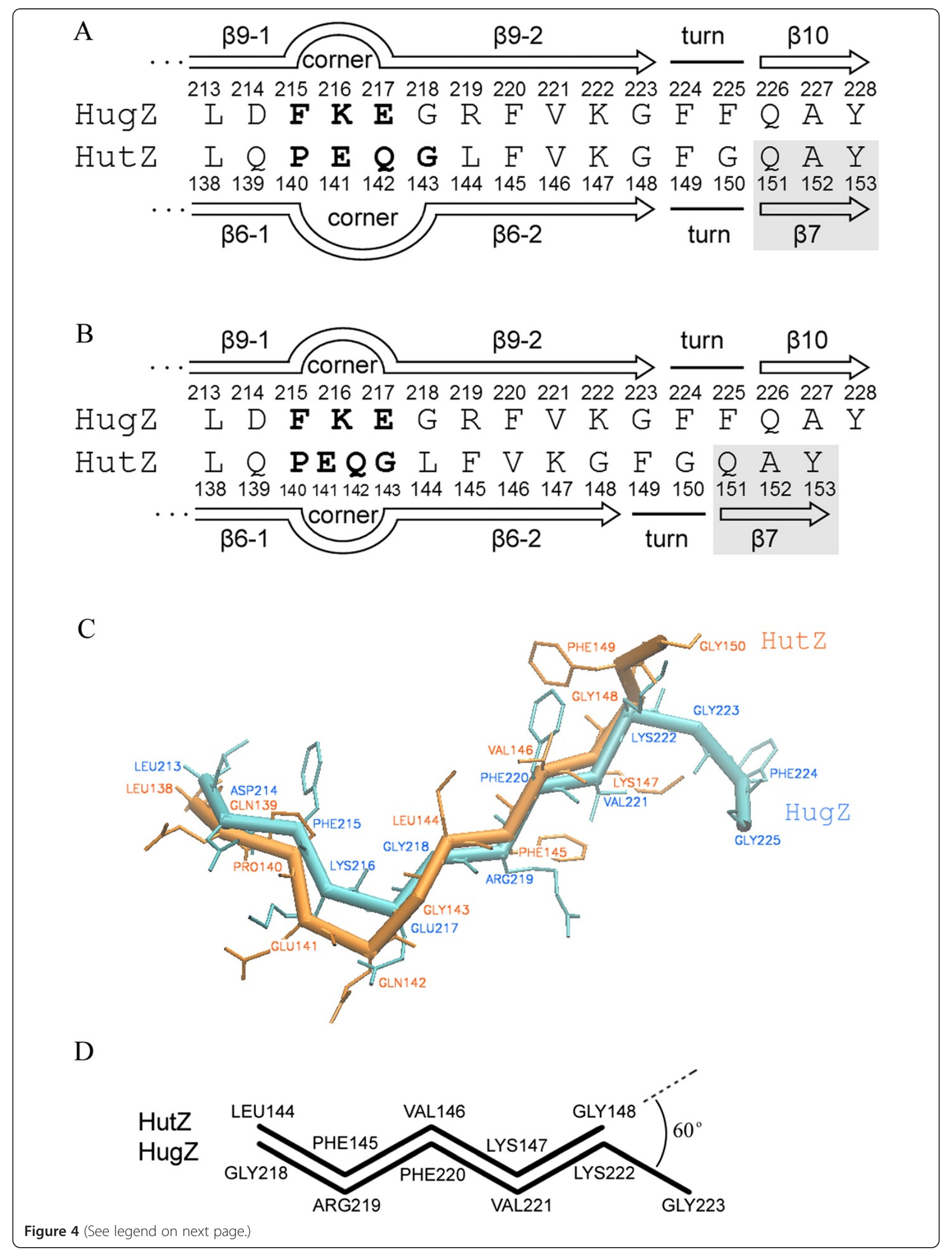


(See figure on previous page.)

Figure 4 Sequence and structural comparisons of HutZ and HugZ structural shift regions. (A) Sequence correspondence based on sequence alignment. Secondary structures are labeled above or below sequences. The shaded region represents the hypothetical $\beta 7$ of HutZ, which does not exist in present crystal structure. (B) Sequence correspondence based on structure superimposition. Different from A, the fourresidue-long corner of HutZ is aligned to three-residue-long corner of HugZ. Secondary structures are labeled above or below sequences. (C) Structural superimposition of HutZ and HugZ. Protein backbones are shown in trace representation with side chains in bond. (D) C-terminals of main chains of $\beta 6-2$ and $\beta 9-2$ point in different directions forming a $60^{\circ}$ angle.

bonds were detected, whereas in the three-residue-long corner of HugZ, two consecutive hydrogen bonds existed on $\mathrm{Lys}^{216}$ and $\mathrm{Glu}^{217}$. In this vein, after both corner regions, the correspondence between the hydrogen bond providing residues on $\beta 6-2$ and $\beta 9-2$ were not in line with that of their sequence alignment (Additional file 5. C). There was a mismatch of one amino acid residue.

Due to this structural mismatch, the side chains of all residues involved in this region pointed to opposite directions in HutZ and HugZ (Figure 4C). The side chain of Phe ${ }^{149}$ in HutZ protruded into a hydrophobic pocket formed by $\mathrm{Ile}^{18}$, $\mathrm{Phe}^{21}$, $\mathrm{Lys}^{91}$ and $\mathrm{Leu}^{93}$, whereas the side chain of corresponding residue Phe ${ }^{224}$ in HugZ turns to another hydrophobic pocket on the opposite side (Figure 5). Starting from this pair of residues, the conformations of the succeeding residues become significantly different between HutZ and HugZ.

\section{Structural modeling of HutZ and its variants}

Anfinsen's dogma [19] states that a protein's native structure is determined by its amino acid sequence and is a stable and kinetically accessible minimum of free energy. Accordingly, the local structural divergence between HutZ and HugZ should result from certain amino acid diversities. Therefore five homologous models for HutZ and its variants were constructed, and their rationalities were assessed to determine their essential residues. The compatibility of every amino acid with its conformation was assessed by ProQres, but the focus was on the $\beta 6$ of HutZ and the corresponding regions in other models, where the structural mismatch occured.

The first model (M1) was generated for the native sequence of HutZ using the HugZ's structure as the template. Thus, M1 possessed HutZ's sequence and did not show a structural mismatch with HugZ. Structural evaluations showed that both crystal structures received higher scores than M1 (Figure 6). This result indicated that the present crystal structure, which possessed a structural mismatch to HugZ, better fitted the HutZ protein sequence than M1, which matched well the structure of HugZ; namely, the structural mismatch of the present crystal structure was reasonable. Simultaneously, the structure of HugZ also well adapted to the sequence of HugZ.

Next, the goal was to identify which residue was the main contributor to HutZ's structural shift. From the

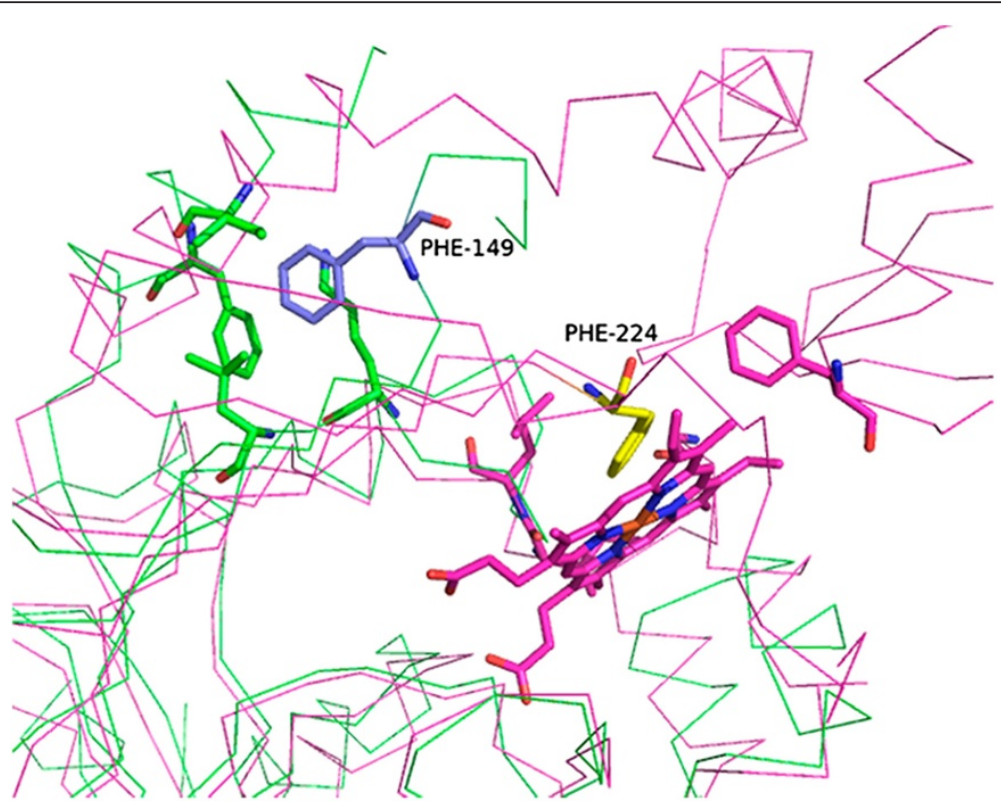

Figure 5 Conformational comparison between Phe ${ }^{149}$ of HutZ and the corresponding residue Phe ${ }^{224}$ of HugZ. HutZ is displayed in green ribbon and HugZ in magenta ribbon. Phe ${ }^{149}$ is labeled as blue stick, and its side chain points to hydrophobic pocket formed by residues in green. $\mathrm{Phe}^{224}$ is in yellow stick, and its side chain points in opposite direction. 


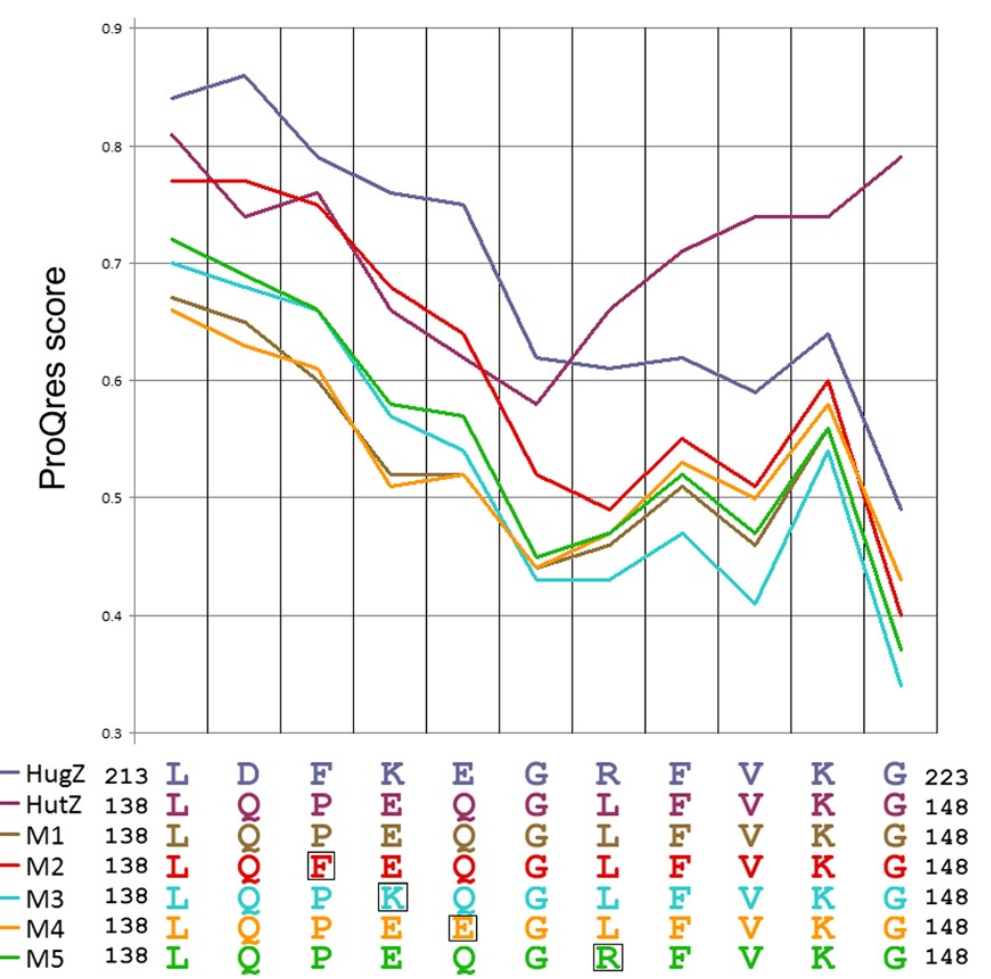

Figure 6 Structure evaluations of crystal structures and models. ProQres score ranged from 0 for a random prediction to 1 for a perfect prediction. Boxed residues are single mutations in models.

beginning of the corner region (Pro ${ }^{140}$ ), where the structural mismatch begins, to $\operatorname{Tyr}^{153}$ of HutZ, there were a total of four different residue pairs between HutZ and HugZ (Figure 4A). These residues were very likely responsible for the structural shift and, thus, four single point variants (P140F, E141K, Q142E and L144R) for HutZ were designed and four models (M2-M5) generated for them, respectively (Figure 6). The mutated residues in HutZ were simply replaced with the corresponding residues of HugZ, and, as with M1, all variant models used the HugZ structure as a template, such that all of them were aligned with HugZ in one-to-one correspondence both on the sequence and structural levels. Structural evaluations of these models suggested three things (Figure 6). First, the two crystal structures were most favored by their sequences, as they generally received higher scores than the homologous models. Second, the score curves of the HugZ crystal structure and all homologous models exhibited similar shapes, which were obviously different from the HutZ crystal structure, because all models were constructed using the HugZ crystal structure as a template. Last, among all homologous models, M2 was evaluated as the best, while M1, M3, M4 and M5 were close to one another, suggesting that the sequence of the variant P140F better fitted the HugZ structure than those of other variants. In other words, although the residues Pro ${ }^{140}, \mathrm{Glu}^{141}, \mathrm{Gln}^{142}$ and
Leu ${ }^{144}$ may together have contributed to the formation of HutZ's structural shift, Pro ${ }^{140}$ played a more important role than other residues. Moreover, $\mathrm{Pro}^{140}$ was located at the very beginning of the corner region, where the structural shift also began, underlining the role of Pro ${ }^{140}$ in the local folding of HutZ $\beta 6$.

\section{Discussion}

HutZ, a unique heme storage protein identified in $V$. cholerae, is necessary for optimal heme utilization $[11,20]$, but no heme oxygenase activity has been detected for HutZ. The crystal structure of the heme oxygenase HugZ shows that there are two symmetric active sites located at the HugZ dimmer interface, formed by the $C$-terminal region $(\beta 8-\beta 11$ and the $C$ terminal loop) from one monomer and $\alpha 7$ from the other [13]. The C-terminal loop functions as a flexible portion of the active site, which is supposed to keep the substrate heme molecule in proper conformation for the heme oxygenase activity as well as to close off the active pocket [13]. In the HutZ structure, the homologous heme-binding pocket involved $\beta 5$ and $\beta 6$, which corresponded with $\beta 8$ and $\beta 9$ in HugZ. The structure from $\beta 7$ to the C-terminal loop, which corresponded to $\beta 10, \beta 11$ and the C-terminal loop in HugZ, was truncated in the present structure. Thus, it was unclear whether the missing portion participated in the heme-binding pocket 
or it resulted in the lack of HutZ enzymic activity. His ${ }^{170}$ from the C-terminal of HutZ, corresponds to the fully conserved $\mathrm{His}^{245}$ in HugZ that is responsible for the heme iron atom coordination [13]. Notably here, HutZ's His ${ }^{170}$ did not contribute to iron coordination and enzymatic activity in HutZ as, when mutant $\mathrm{H} 170 \mathrm{~A}$ was reconstituted with heme, the Soret peak did not change in comparison with the native HutZ-heme complex (data not shown). This study of the structural mismatch between HutZ and HugZ provided an important clue for resolving this issue. Because the corner of $\beta 6$ of HutZ includes one more amino acid residue than that of HugZ, HutZ's $\beta 6-2$ (five residues) ends one amino acid earlier than HugZ's $\beta 9-2$ (six residues). It is known that the torsion angle of $\mathrm{N}-\mathrm{C} \alpha-$ $\mathrm{C}-\mathrm{N}$ in the backbone of a $\beta$-strand is about $120^{\circ}$. Therefore, the main chains of the end residues of the two $\beta$-strands ( $36-2$ and $\beta 9-2)$ point in different directions to form a $60^{\circ}$ angle (Figure 4D). This direction deviation led to an absolute mismatch between the structures of HutZ ( $\mathrm{Phe}^{149}$ and Gly $^{150}$ ) and HugZ (Gly ${ }^{223}$ and $\mathrm{Phe}^{224}$ ) \(Figure 4C), and might have led to even larger structural differences in their C-terminal regions (missing in the present structure). In this regard, it was speculated that the C-terminal loop, which is essential for HugZ enzymatic activity, might have turned away from the heme-binding pocket of Hut $Z$ and yielded the pocket more exposed than that of HugZ. This might then have resulted in low heme-binding affinity and a deficiency in HutZ enzymatic activity. In addition, the possibility was excluded here that truncating the protein resulted in a structural shift of $\beta 6-2$ in HutZ for three reasons. First, HutZ was truncated here because of the twinning and fragile crystals of the full length protein. If the complete HutZ structure matched HugZ at $\beta 6-2$ and the C-terminal loop, good full length crystals would have been obtained, as have been attained by $\mathrm{Hu}$ and coworkers [13]. Second, an extensive hydrogen bond network connected two parallel $\beta$-stands and maintained their stable conformations. Truncating the residues that follow $\beta 6-2$ might not have provided enough energy to destroy the normal hydrogen bonding and rearrange them. And third, the hypothetical HutZ structure (M1) that matches HugZ at $\beta 6-2$ evaluated even more poorly than the present truncated HutZ structure.

\section{Methods}

\section{Protein expression, purification and site-directed mutagenesis}

The hutZ gene was amplified from genomic DNA of $V$. cholerae and subcloned into the pET21b expression vector (Novagen, EMD Biosciences, Inc., Darmstadt, DE) between the NdeI and XhoI restriction cut sites. The N-terminal and C-terminal regions (residues 1-12 and 151-176, respectively) were removed during cloning, resulting in a construct of 13-150-His tagged fusion protein for further expression.

BL21 (DE3) cells containing plasmids for the recombinant HutZ protein were grown in L Broth media supplemented with $100 \mu \mathrm{g} / \mathrm{mL}$ ampicillin. Once the culture attained an $\mathrm{OD}_{600}$ of 1.0 , the incubation temperature was decreased to $15^{\circ} \mathrm{C}$, and protein expression induced by adding isopropyl $\beta$-D-1-thiogalactopyranoside to a final concentration of $0.10 \mathrm{mM}$. After $8 \mathrm{~h}$ of expression, the cells were harvested by centrifugation and the pellet resuspended in lysis buffer $(15 \mathrm{mM}$ Tris- $\mathrm{HCl}, \mathrm{pH}$ 8.0, $100 \mathrm{mM} \mathrm{NaCl}$, and $1 \mathrm{mM}$ phenyl methane sulfonyl fluoride), and lysed by sonication. Cell debris was then removed by centrifugation at $28500 \times \mathrm{g}$ for $45 \mathrm{~min}$. The resulting soluble fractions containing recombinant protein HutZ was loaded onto a Ni-chelating Sepharose affinity column (GE Healthcare, Buckinghamshire, UK) equilibrated with lysis buffer. The affinity column was washed extensively with lysis buffer and all proteins eluted with elution buffer (20 mM Tris- $\mathrm{HCl}, \mathrm{pH} 8.0,50 \mathrm{mM}$ $\mathrm{NaCl}$ and $200 \mathrm{mM}$ imidazole). The elution protein was further purified using an ion-exchange column (Source 15Q, GE Healthcare), conditioned with equilibration buffer (25 mM Tris-HCl, pH 8.0, 3 mM DTT), and eluted using a linear $150 \mathrm{~mL}$ gradient of $0-0.5 \mathrm{M} \mathrm{NaCl}$. Finally, HutZ protein was purified using size exclusion chromatography (Superdex-200, GE Healthcare). Fractions were pooled

\section{Table 1 Statistics of crystallographic analysis}

\begin{tabular}{|c|c|}
\hline Data collection & \\
\hline Space group & $\mathrm{P} 4_{1}$ \\
\hline unit cell $(\AA ̊)$ & $a=b=80.1 \quad c=125.8$ \\
\hline Resolution $(\AA)$ & $50-2.15$ \\
\hline Completeness & $95.5(95.9)^{\mathrm{a}}$ \\
\hline Redundancy & $10.0(7.8)$ \\
\hline $\mathrm{I} / \sigma(\mathrm{I})$ & $24.5(7.2)$ \\
\hline Rsym $(\%)^{b}$ & $10.4(47.4)$ \\
\hline \multicolumn{2}{|l|}{ Refinement } \\
\hline Resolution & $50-2.0$ \\
\hline Rwork/Rfree $(\%)^{c}$ & 19.73/23. 51 \\
\hline \multicolumn{2}{|l|}{ RMSD } \\
\hline Bond lengths $(\AA)$ & 0.007 \\
\hline Bond angles $\left(^{\circ}\right)$ & 1.070 \\
\hline \multicolumn{2}{|l|}{ Ramachandran plot (\%) $^{d}$} \\
\hline Most favored (\%) & 92.9 \\
\hline Additionally allowed (\%) & 6.9 \\
\hline Generously allowed (\%) & 0.2 \\
\hline Disallowed (\%) & 0.0 \\
\hline
\end{tabular}

${ }^{a}$ Values in parentheses are for reflections in the highest resolution shell.

${ }^{\mathrm{b}} R_{\text {sym }}=\sum_{h k l} \sum_{i}\left|l(h k)_{i}-<l(h k l)>\right| / \sum_{h k l} \sum l<l(h k)_{i}>$ over $i$ observations.

c Value of $R_{\text {free }}$ for $3.91 \%$ of randomly selected reflections excluded from refinement.

${ }^{d}$ As defined in PROCHECK. 
according to protein purity monitored by SDS-PAGE, and the final protein concentration at $12 \mathrm{mg} / \mathrm{mL}$. All mutant proteins were purified using the same procedure.

\section{Crystallization and data collection}

HutZ was crystallized using the sitting drop vapor diffusion method at $20^{\circ} \mathrm{C}$ by mixing equal volumes of protein with reservoir solution containing $1.6 \mathrm{M} \mathrm{Na} / \mathrm{K}$ phosphate and 0.1 M HEPES at $\mathrm{pH}$ 7.5. Because the crystals of full length HutZ were twinning and fragile and could not be used to obtain high resolution diffraction data, the protein was truncated to amino acid residues 13-150 to grow high quality crystals, which appeared about seven to ten $d$ and reached full size in two wk. Diffraction data were collected at the Shanghai Sychrotron Radiation Facility beamline BL17U1. To prevent radiation damage, crystals were transferred to a cryoprotectant buffer containing 15\% glycerol (v/v) plus reservoir buffer and then flash-cooled using a nitrogen stream, with the temperature around the crystals maintained at $100 \mathrm{~K}$ throughout data collection. Data sets were processed using the HKL2000 software suite [21]. The crystals belonged to the space group $\mathrm{P} 4_{1}$ with four macromolecules in an asymmetric unit, with unit cell dimensions of $\mathrm{a}=\mathrm{b}=80.1 \AA$ and $\mathrm{c}=125.8 \AA$.

\section{Structure determination}

HutZ's structure was determined by molecular replacement with PHASER from CCP4i software, using the search model Alr5027 (PDB code: 1VL7) from Nostoc sp. with sequence identity of $43 \%$. The atomic model was built using COOT [22] and refined using PHENIX [23]. Data collection and structure refinement statistics are shown in Table 1. All molecular graphics figures were generated with PyMol (http://www.pymol.org).

\section{Docking and homology modeling}

AutoDock 4.2 [24] was used to perform flexible docking in the HutZ-heme complex. The heme was restricted within a grid box $(50 \times 50 \times 30$ points in dimension and $0.375 \AA$ spacing) that enveloped the HutZ binding cleft, which was determined through similarities with the known crystal structure of the HugZ-heme complex. Docking searches were executed using the Lamarckian genetic algorithm and a maximum number of 25,000,000 energy evaluations. After docking searches were finished, the first-ranked model, based on binding energy, was selected as the final result from 50 candidate solutions.

A series of homologous models of HutZ and its variants were generated using the MODELLER 9.9 program package [25] with HugZ (PDB code: 3GAS) as a template. The input files for each target sequence were a pairwise sequence alignment (template and target) and the coordinate file of the template. The number of output models for each target was set to five, and other MODELLER options at default. The qualities of all resulting models were evaluated with ProQres [26], a neuralnetwork based local protein model quality predictor, which analyzes the compatibility of every amino acid residue with its conformation in a three-dimensional model.

\section{Conclusions}

We determined the crystal structure of HutZ from $V$. cholerae at $2.0 \AA$ resolution and compared the structure with that of its homologous protein HugZ. The structural mismatch between HutZ and HugZ presented a rare case in which the structural alignment was not in accordance with the sequence alignment for two highly similar proteins (structure RMSD $=1.645 \AA$ and sequence identity = $35 \%)$. This observation suggested a potential hazard in the assumed accuracy of template selection of the traditional homology modeling method. If a homologous model of HutZ is constructed using the default sequence alignment with HugZ and the HugZ structure as a template, the resulting model will be inaccurate.

\section{Additional files}

\begin{abstract}
Additional file 1: Chromatographic analysis of HutZ and two unpublished proteins $\mathbf{A}$ and $\mathbf{B}$. Both proteins $A$ and $B$ exist as monomer in solution. The elution volume for $A(34.7 \mathrm{KD})$ and $B(17.5 \mathrm{KD})$ are $14.9 \mathrm{~mL}$ and $17.9 \mathrm{~mL}$, respectively. The elution volume of HutZ (15 KD) was $15.7 \mathrm{~mL}$. These results indicate that HutZ exists as dimer.
\end{abstract}

Additional file 2: Greek key topology of $\beta$-barrels of HutZ and HugZ.

Additional file 3: Sequence alignment of HutZ with its homologous proteins. Vc, Vibrio cholerae HutZ; As, Aliivibrio salmonicida HuvZ; Pss, Photobacterium sp. SKA34 HugZ; Va, Vibrio alginolyticus protein V12G0106051; Ah, Aeromonas hydrophila HutZ; Hi, H. influenza protein HI0854; Cj, C. jejuni ChuZ; Hp, H. pylori HugZ. Secondary structures of Vc-HutZ are schematically represented above the sequences. Residues from HutZ that coordinate the iron atom in heme are labeled with asterisks at the top; residues stabilizing the heme molecule in $\mathrm{HutZ}$ are labeled with squares at the top; residues from $\mathrm{HugZ}$ coordinating the iron atom in heme are labeled with rhombus at the bottom; residues from HugZ involved in stabilization of heme molecule are labeled with triangles at the bottom. Strictly conserved residues are marked with red background. Similar residues are shown in red color.

Additional file 4: An Fo Fc omit map calculated at 2.0 Å resolution (contoured at 1.0 sigma) for the four residues making up the $\beta 6$ corner of HutZ. The electron density map shown in gray well matches the corresponding structure, indicating the reliability of this structure.

Additional file 5: Different hydrogen bonding patterns in HutZ $\beta 6$ and HugZ $\beta$ 9. (A) Hydrogen bonding patterns in HutZ $\beta 6$. Blue sphere: nitrogen atom; red sphere: oxygen atom; green dotted line: hydrogen bond. (B) Hydrogen bonding patterns in HugZ $\beta$ 9. (C) Sequence correspondence based on sequence alignment. Secondary structures are labeled above or under the sequences. Hydrogen bond providing residues are in bold. From the corners up, the hydrogen bonding patterns become irregular. In $\beta 6-2$ and $\beta 9-2$, the hydrogen bond providing residues mismatch by one amino acid residue. 


\section{Accession codes}

The atomic coordinates and structure factors have been deposited in the Protein Data Bank (http://www.rcsb.org/pdb/) with the accession code 3TGV.

\section{Authors' contributions}

LG designed the study. XL and JG participated in the design of the study, carried out all experiments in molecular biology, protein chemistry, structure refinement, and drafted the manuscript. JG and TW performed the bioinformatic analysis. ZW and QD synthesized proteins. DZ and YH collected the $\mathrm{X}$-ray data. $\mathrm{XL}$ and JG should be regarded as joint first authors. All authors read and approved the final manuscript.

\section{Acknowledgement}

The genomic DNA of Vibrio cholerae is a gift from Prof. Bonnie Bassler. The authors thank the staff at beamline BL17u1 at the Shanghai Synchrotron Radiation facility for support with data collection. This work was supported by State Key Laboratory of Microbial Technology (SDU), the Grant of Hi-Tech Research and Development Program of China (2006AA02A324) and Promotive Research Fund for Excellent Young and Middle-aged Scientists of Shandong Province (BS2012SW006).

\section{Author details}

'State Key Laboratory of Microbial Technology, School of Life Sciences, Shandong University, Jinan 250100, China. ${ }^{2}$ Cancer Research Center, School of Medicine, Shandong University, Jinan 250012, China. ${ }^{3}$ College of Life Sciences, Hebei University, Baoding 071002, China.

Received: 15 April 2012 Accepted: 24 September 2012 Published: 26 September 2012

\section{References}

1. Wyckoff EE, Mey AR, Payne SM: Iron acquisition in vibrio cholerae. Biometals 2007, 20(3-4):405-416.

2. Griffiths GL, Sigel SP, Payne SM, Neilands JB: vibriobactin, a siderophore from vibrio cholerae. J Biol Chem 1984, 259(1):383-385.

3. Seliger SS, Mey AR, Valle AM, Payne SM: The two TonB systems of vibrio cholerae: redundant and specific functions. Mol Microbiol 2001, 39(3):801-812.

4. Mey AR, Wyckoff EE, Oglesby AG, Rab E, Taylor RK, Payne SM: Identification of the vibrio cholerae enterobactin receptors VctA and IrgA: IrgA is not required for virulence. Infect Immun 2002, 70(7):3419-3426.

5. Wyckoff EE, Valle AM, Smith SL, Payne SM: A multifunctional ATP-binding cassette transporter system from vibrio cholerae transports vibriobactin and enterobactin. J Bacteriol 1999, 181(24):7588-7596.

6. Rogers MB, Sexton JA, DeCastro GJ, Calderwood SB: Identification of an operon required for ferrichrome iron utilization in vibrio cholerae. J Bacteriol 2000, 182(8):2350-2353.

7. Stoebner JA, Payne SM: Iron-regulated hemolysin production and utilization of heme and hemoglobin by vibrio cholerae. Infect Immun 1988, 56(11):2891-2895.

8. Henderson DP, Payne SM: Cloning and characterization of the vibrio cholerae genes encoding the utilization of iron from haemin and haemoglobin. Mol Microbiol 1993, 7(3):461-469.

9. Occhino DA, Wyckoff EE, Henderson DP, Wrona TJ, Payne SM: vibrio cholerae iron transport: haem transport genes are linked to one of two sets of tonB, exbB, exbD genes. Mol Microbiol 1998, 29(6):1493-1507.

10. Wheeler DL, Barrett T, Benson DA, Bryant SH, Canese K, Chetvernin V, Church DM, Dicuccio M, Edgar R, Federhen S, et al: Database resources of the national center for biotechnology information. Nucleic Acids Res 2008, 36(Database issue):D13-D21.

11. Wyckoff EE, Schmitt M, Wilks A, Payne SM: HutZ is required for efficient heme utilization in vibrio cholerae. J Bacteriol 2004, 186(13):4142-4151.

12. Guo Y, Guo G, Mao X, Zhang W, Xiao J, Tong W, Liu T, Xiao B, Liu X, Feng Y, et al: Functional identification of HugZ, a heme oxygenase from helicobacter pylori. BMC Microbiol 2008, 8:226.

13. Hu Y, Jiang F, Guo Y, Shen X, Zhang Y, Zhang R, Guo G, Mao X, Zou Q, Wang DC: Crystal structure of HugZ, a novel heme oxygenase from helicobacter pylori. J Biol Chem 2011, 286(2):1537-1544.

14. Zhang R, Zhang J, Guo G, Mao X, Tong W, Zhang Y, Wang DC, Hu Y, Zou Q: Crystal structure of Campylobacter jejuni ChuZ: a split-barrel family heme oxygenase with a novel heme-binding mode. Biochem Biophys Res Commun 2011, 415(1):82-87.
15. Hirotsu S, Chu GC, Unno M, Lee DS, Yoshida T, Park SY, Shiro Y, IkedaSaito M: The crystal structures of the ferric and ferrous forms of the heme complex of $\mathrm{HmuO}$, a heme oxygenase of corynebacterium diphtheriae. J Biol Chem 2004, 279(12):11937-11947.

16. Zhu W, Wilks A, Stojiljkovic I: Degradation of heme in gram-negative bacteria: the product of the hemO gene of Neisseriae is a heme oxygenase. J Bacteriol 2000, 182(23):6783-6790.

17. Skaar EP, Gaspar AH, Schneewind O: Bacillus anthracis IsdG, a hemedegrading monooxygenase. J Bacteriol 2006, 188(3):1071-1080.

18. Suits MD, Pal GP, Nakatsu K, Matte A, Cygler M, Jia Z: Identification of an Escherichia coli 0157:H7 heme oxygenase with tandem functional repeats. Proc Natl Acad Sci U S A 2005, 102(47):16955-16960.

19. Anfinsen CB: Principles that govern the folding of protein chains. Science 1973, 181(96):223-230

20. Wilks A: The ShuS protein of Shigella dysenteriae is a heme-sequestering protein that also binds DNA. Arch Biochem Biophys 2001, 387(1):137-142.

21. Otwinowski Z, Minor W: Processing of $x$-ray diffraction data collected in oscillation mode. Methods Enzymol 1997, 276:307-326.

22. Emsley P, Cowtan K: Coot: model-building tools for molecular graphics. Acta Crystallogr D: Biol Crystallogr 2004, 60(Pt 12 Pt 1):2126-2132.

23. Adams PD, Grosse-Kunstleve RW, Hung LW, loerger TR, McCoy AJ, Moriarty NW, Read RJ, Sacchettini JC, Sauter NK, Terwilliger TC: PHENIX: building new software for automated crystallographic structure determination. Acta Crystallogr D: Biol Crystallogr 2002, 58(Pt 11):1948-1954.

24. Morris GM, Huey R, Lindstrom W, Sanner MF, Belew RK, Goodsell DS, Olson AJ: AutoDock4 and AutoDockTools4: Automated docking with selective receptor flexibility. J Comput Chem 2009, 30(16):2785-2791.

25. Eswar N, Webb B, Marti-Renom MA, Madhusudhan MS, Eramian D, Shen MY Pieper U, Sali A: Comparative protein structure modeling using Modeller. Curr Protoc Bioinformatics 2006, Chapter 5:Unit 56.

26. Wallner B, Elofsson A: Identification of correct regions in protein models using structural, alignment, and consensus information. Protein Sci 2006 15(4):900-913.

doi:10.1186/1472-6807-12-23

Cite this article as: Liu et al:: Crystal structure of HutZ, a heme storage protein from Vibrio cholerae: A structural mismatch observed in the region of high sequence conservation. BMC Structural Biology 2012 12:23.

\section{Submit your next manuscript to BioMed Central and take full advantage of:}

- Convenient online submission

- Thorough peer review

- No space constraints or color figure charges

- Immediate publication on acceptance

- Inclusion in PubMed, CAS, Scopus and Google Scholar

- Research which is freely available for redistribution 